\title{
Design and Development of Intelligent ECG Monitoring System Based on ARM
}

\author{
Yang Bao-liang ${ }^{1, a}$, Gao-Xiang ${ }^{1, b}$, Li-Peng ${ }^{1, c_{*}}$ Ye Jia-yong ${ }^{2, d}$ \\ 1Chongqing University of Arts and Sciences Chongqing 402160 P. R. China \\ 2Chongqing Industrial vocational school Chongqing 402160 P. R. China \\ a Email:327132567@qq.com \\ b1140354190@qq.com \\ C*Email: lip663073@163.com \\ d1047982619@qq.com
}

Keywords: ARM; ECG signal; STM32; portable; USB

Abstract. Based on the ECG monitoring instrument and ECG signal research, design a series of ARM chip based on STM32 as the core of the portable home ECG detection equipment. The device with three electrodes of ECG signal acquisition, due to weak signal acquisition processor cannot directly handle the ECG acquisition circuit, but also on the ECG signal amplification, filtering and level uplift is sent to the ARM processor, the ARM processor on the received analog signals through the built-in AD discretization, and then the analysis and processing of data real time display on the LCD screen, ECG, heart rate and various analytical results, but also can through the USB interface to a detailed analysis of the data transmission to the host computer. Tests prove that, the system has the advantages of simple structure, stable and reliable work, the ECG can get a clear, stable and biological signals can reflect the real ECG features of human body, according to expert system comes with the system, can to the condition estimation, simultaneously has the advantages of simple operation, low price, easy to carry, has certain market promotion value.

\section{Introduction}

With the increasing number of aging Chinese, health care of the elderly has aroused great concern in the community, but in China because of imbalance of population and medical equipment, large hospitals are overcrowded every day and most of the time is spent in the queue. Most of the rural areas have no access to medical equipment monitoring, for diseases cannot be timely discovered and best treatment opportunities are missed. Heart disease is a common multiple chronic diseases, and has long been a threat to human health. The timely detection and prevention in reducing cardiovascular disease is very important. The electrocardiogram can guarantee the timely detection of this kind of disease, but due to the current ECG machine volume and high price limit, patients can't get the real-time monitoring which is extremely unfavorable for the patient's diagnosis and treatment. Portable ECG monitoring system we design has the advantages of being simple structure, small volume and easy to carry, and can acquire the ECG signal whenever and wherever possible with real-time analysis of such ECG signal. Through the built-in large capacity memory and time system, the system can carry out real-time monitoring of patients for a long time, and record and store the ECG data of patients. At the same time, the system also can transmit data through USB interface with PC for further analysis ${ }^{\text {[1] }}$.

\section{The structure of the ECG monitoring system}

The traditional ECG measurement, generally uses the five lead structure, namely by clicking on the link in the human left arm, left ankle and the anterior chest, then measurement ${ }^{\text {'2l }}$. This method is accurate, but the need for many connecting wire leads to the long time consuming measurement. At the same time, the complex operation can not make ECG measurement as easy as temperature 
measurement .In order to solve the problems above; we use the ST Company's production of a high performance and low price of the ARM series STM32 processor to design a small size, light weight and powerful portable ECG monitoring system. This system mainly includes the STM32 core processor, ECG signal acquisition and processing module, power module, keyboard circuit, USB data communication module, data storage module, liquid crystal display module. With ECG signal acquisition and processing module and ECG signal pretreatment module, ECG signal is acquired by the electrode and sent to ECG acquisition circuit which afterwards goes through the preamplifier, main amplifier, low pass filter, level elevation. Then the processed signal is sent to the STM32 core processor. In order to suppress the interference signal, the driven right leg circuit is introduced to improve the anti-interference performance of the system. STM32 core processor first conduct the AD conversion of the ECG signal which is sent by the acquisition module with internal ADC, and then analyzed the discrete data. The liquid crystal display module is responsible for ECG and ECG information display. Data memory is for the user's information storage of each phase, which will provide the basic data for the future effective analysis. The USB communication interface can send ECG data of the system to the host computer through the reliable USB transmission for further analysis.

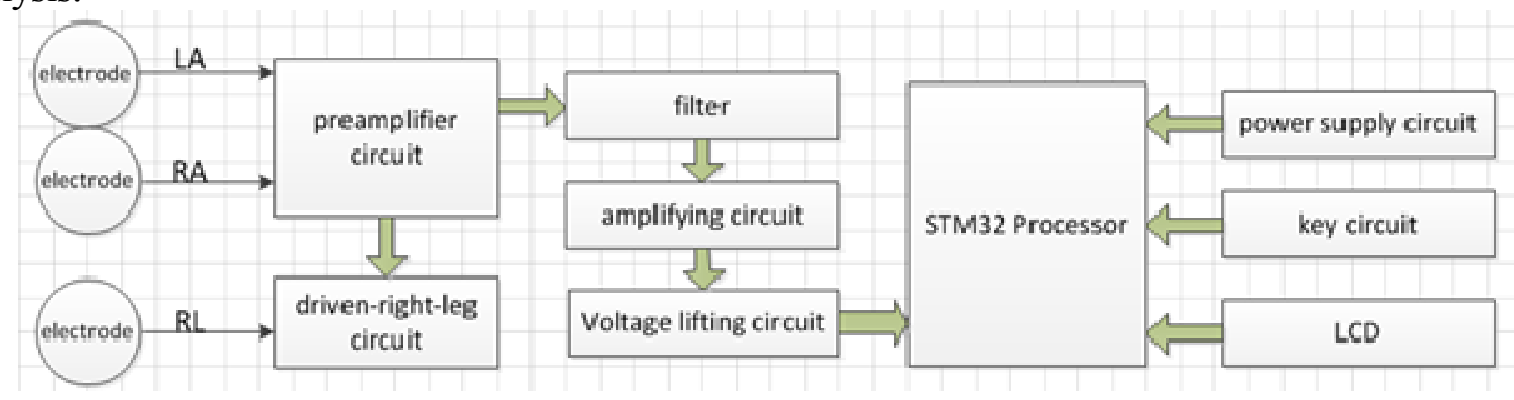

Figure 1 the structure of the ECG monitoring system

\section{The hardware circuit design of the systems.}

The system mainly consists of ECG acquisition and pre-processing circuit, STM32 minimum system circuit, data communication circuit, liquid crystal display circuit and data storage circuit. Here we mainly introduce the core circuit of STM32 and ECG collecting circuit.

\section{ECG signal acquisition and processing circuit}

ECG signal acquired from the surface of the body is very weak, generally only $0.1-3 \mathrm{mV}$, and has the characteristics of instability, low frequency characteristic, randomness, and very vulnerable to the interference of external environment ${ }^{[2]}$. The interference of ECG signal mainly includes the frequency interference, high frequency electromagnetic field, polarization interference and the interference of measuring equipment. According to the ECG features above, we are design an ECG acquisition circuit in accordance with the standard I lead. Structure diagram is shown in figure 2.

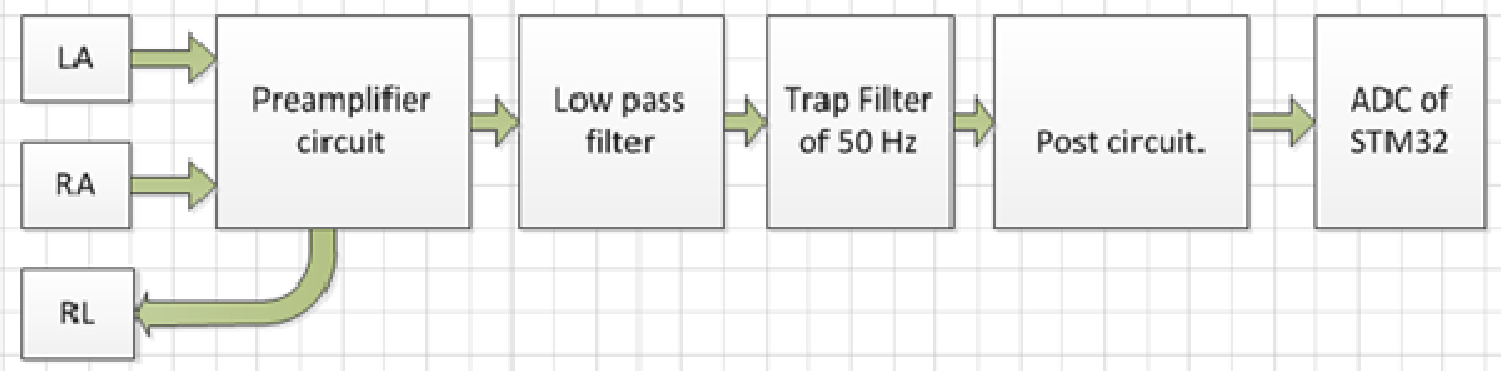

Figure 2 ECG signal acquisition and processing unit

The ECG signal obtained by the heart electrodes is sent into the preamplifier circuit of amplifier and high performance differential amplifier circuit can suppress common mode interference signal. At the 
same time, the quality of signal acquisition is improved through the right leg circuit suppressing common mode interference and $50 \mathrm{~Hz}$ interference. After the preamplifier circuit of ECG signal amplification, the preliminary processed ECG signal is sent into the cut-off frequency of $0.5-100 \mathrm{~Hz}$ high pass and low pass filter circuit. Then input the signal to main amplifier to realize the 100 times amplification, which means the signal is amplified to a range of 0.08-2.7 V. In order to eliminate the noise in the signal amplification, and filter the signal of $50 \mathrm{~Hz}$ frequency signal, the amplified signal go through $50 \mathrm{~Hz}$ notch, and then through the low pass filter circuit, so as to get the clear waveform. The ECG signal is AC signal and acquisition input range of $\mathrm{A} / \mathrm{D}$ microcontroller is $0-3.3 \mathrm{~V}$, so the signal level needs conversion and to be uplifted the to the MCU sampling range of analog voltage for the transformation of $\mathrm{A} / \mathrm{D}$, which thus meet the analysis, the storage and transmission requirements of the embedded system .

\section{The preamplifier circuit design}

Because the human ECG signal is very weak with strong interference and noise, and there are different polarization voltages, the primary amplifier must have high input impedance, high common mode rejection ratio, low noise, low drift, small nonlinearity, suitable bandwidth and dynamic range performance. Design by adopting the differential amplifier circuit, as shown in Figure 3, and choose instrumentation amplifier AD620 chip. Because the gain of AD620 is affected by follow formula : $\mathrm{G}=1+(\mathrm{R} 1+\mathrm{R} 2) / \mathrm{R} 3$, so the value of $\mathrm{R} 21$ and $\mathrm{R} 22$ are $27 \mathrm{~K}$, the value of $\mathrm{R} 23 \mathrm{is} 6.2 \mathrm{~K}$, the value of $\mathrm{C} 21$ is $39 \mathrm{pF}$, the value of $\mathrm{C} 22$ is $200 \mathrm{pF}$, the value of C23is 39Pf, Therefore The results for the pre amplification is 9.7

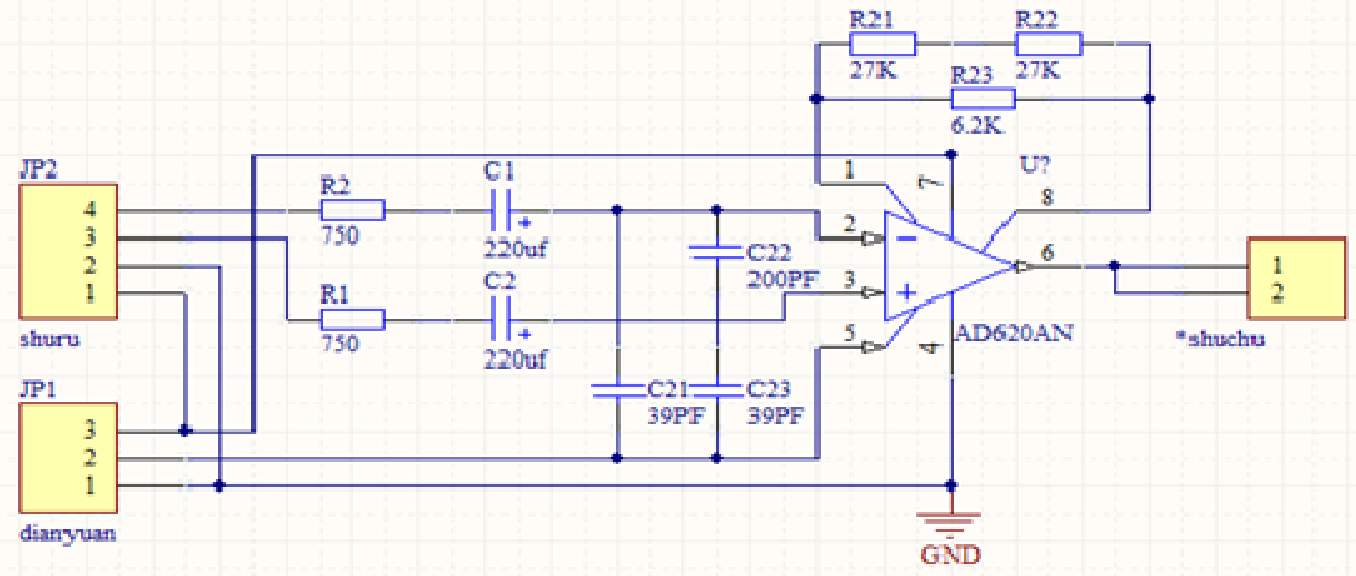

Figure 3 The preamplifier circuit

\section{Software design of the ECG monitoring system}

As we knows that you cannot make bricks without straw.so we development the software of embedded system with the good SDK, therefore we use is Keil MDK, which is micro controller software development platform by a well-known German software company Keil (now part of ARM) and is currently the mainstream ARM kernel microcontroller development tools. Keil provides a complete development plan including the $\mathrm{C}$ compiler, macro assembler, linker, library management and a powerful simulation debugger, and through an integrated development environment (uVision) these functions will be combined together. The software uses the modular design method and the program includes main program and interrupt program, data acquisition and data communication interrupt program. The main program is responsible for the LCD screen and receiving data sent from a user control commands. Data acquisition interrupt program mainly samples ECG signal by $480 \mathrm{HZ}$ the frequency. Data communication interrupt program mainly transmit data through the USB port and store the data. the main program flow chart and the data acquisition program is shown in figure4. 


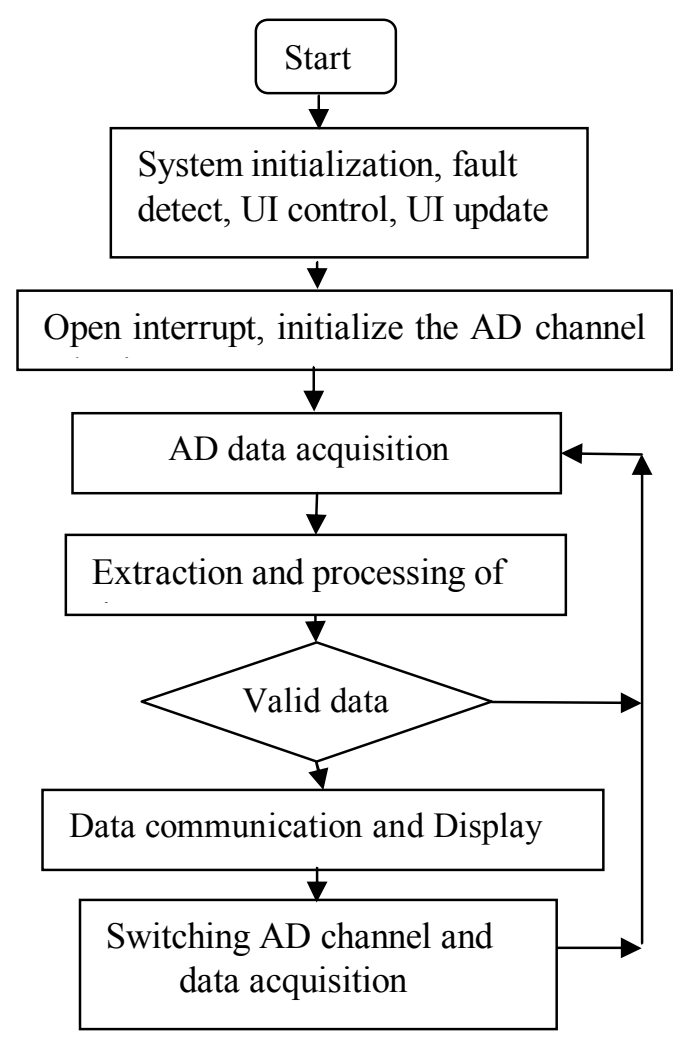

Figure 4 The flow chart of main program

\section{The analysis of the test results}

The electrode ends linked to left and right finger, waveforms are found on LCD as shown in Figure 5 , only a relatively close to the real ECG signal. Seen from the figure liquid crystal display of the waveform, the ECG waveform display appears clear and stable with the obvious characteristics of ECG waveform, which can reflect the actual characteristics of the human body

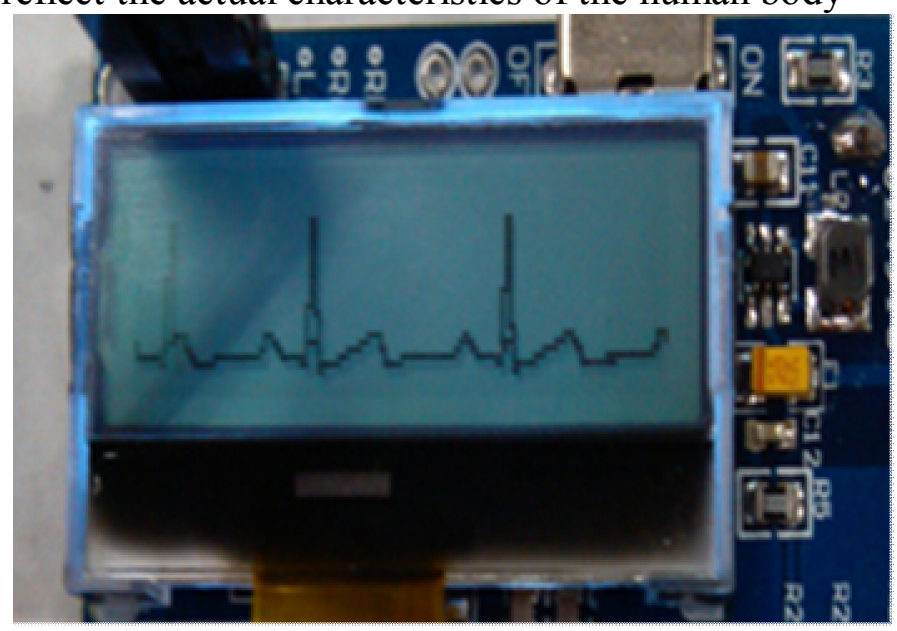

Figure 5 The waveforms of ECG acquisition 


\section{Summary}

STM32 as the main control chip, which contains abundant function module and advanced standard communication interface can complete the acquisition, storage and data communication of ECG signal without external expansion chip Therefore, the ECG instrument has the characteristics of small volume, low power consumption which meet the basic requirements of the portable device. The experimental results show that the system has achieved the anticipated effect.

With the increasing incidence of cardiovascular disease year by year, the portable ECG device has high application value and good market prospects.

\section{Acknowledgements}

This work was supported by the Chongqing University of Arts and Sciences Foundation (Grant No: Z2014DQ06), and Chongqing University of Arts and Sciences Foundation of Student(Grant No: XSKY2014061) .

\section{References}

[1] X.Chao, Z.F.Wu. Portable Electrocardiogram Measurement base on STM32 [J]. Equipment Manufactring Technology .2012(1):57-65.

[2]XIONG Xin,CEN Ren-jing. Research on Recognizing the Interference of Dynamic ECG Signal[J]. China Medical Devices.2010(3):34-36.

[3]Wang Zihong, Wu Baoming. Development of a Wearable Electrocardiogram Monitor with Recognition of Physical Activity Scene[J]. Journal of Biomedical Engineering. 2012(5) :941-947.

[4] LI Zhi-jian,HUANG Guo-ming. Design of ECG signal acquisition circuit [J]. Modern Electronics Technique. 2013(15):146-152.

[5] Chen Yingzhao;Gao Yueming. Design of a portable ECG detecting instrument based on STM32 [J]. Application of Electronic Technique. 2013(9) :18-20.

[5] WU Lizhen,ZHANG Wenchao. Design of Portable ECG Device Based on STM32 [J]. Chinese Journal of Electron Devices. 2009(5) :947-949. 